\title{
Phase-coded pulse aperiodic transmitter coding
}

\author{
I. I. Virtanen ${ }^{1}$, J. Vierinen ${ }^{2}$, and M. S. Lehtinen ${ }^{2}$ \\ ${ }^{1}$ Department of Physics, University of Oulu, P.O. Box 3000, 90014 Oulu, Finland \\ ${ }^{2}$ Sodankylä Geophysical Observatory, 99600, Sodankylä, Finland \\ Received: 16 February 2009 - Accepted: 18 June 2009 - Published: 14 July 2009
}

\begin{abstract}
Both ionospheric and weather radar communities have already adopted the method of transmitting radar pulses in an aperiodic manner when measuring moderately overspread targets. Among the users of the ionospheric radars, this method is called Aperiodic Transmitter Coding (ATC), whereas the weather radar users have adopted the term $\mathrm{Si}$ multaneous Multiple Pulse-Repetition Frequency (SMPRF). When probing the ionosphere at the carrier frequencies of the EISCAT Incoherent Scatter Radar facilities, the range extent of the detectable target is typically of the order of one thousand kilometers - about seven milliseconds - whereas the characteristic correlation time of the scattered signal varies from a few milliseconds in the D-region to only tens of microseconds in the F-region. If one is interested in estimating the scattering autocorrelation function (ACF) at time lags shorter than the F-region correlation time, the D-region must be considered as a moderately overspread target, whereas the F-region is a severely overspread one. Given the technical restrictions of the radar hardware, a combination of ATC and phase-coded long pulses is advantageous for this kind of target. We evaluate such an experiment under infinitely low signal-to-noise ratio (SNR) conditions using lag profile inversion. In addition, a qualitative evaluation under highSNR conditions is performed by analysing simulated data. The results show that an acceptable estimation accuracy and a very good lag resolution in the D-region can be achieved with a pulse length long enough for simultaneous E- and Fregion measurements with a reasonable lag extent. The new experiment design is tested with the EISCAT Troms $\emptyset$ VHF $(224 \mathrm{MHz})$ radar. An example of a full D/E/F-region ACF from the test run is shown at the end of the paper.
\end{abstract}

Keywords. Ionosphere (Instruments and techniques) - Radio science (Ionospheric physics)

Correspondence to: I. I. Virtanen

(ilkka.i.virtanen@oulu.fi)

\section{Introduction}

The simplest design of a radar experiment is to transmit short pulses at equal inter-pulse periods (IPP) between the pulses, i.e. to use a uniform pulse-repetition frequency (PRF). To cover the whole range extent of the target, the IPP must be larger than or equal to the roundtrip time of the signal to and from the furthermost target range. Thus, the range extent of the target sets an upper limit for the PRF. A radar target is called overspread if its scattering spectrum is wider than the largest possible uniform PRF.

Severely overspread radar targets, whose scattering spectrum is much wider than the largest possible PRF, can be sufficiently sampled by only measuring lag values clearly shorter than the signal roundtrip time to and from the target. They can be efficiently measured using long, possibly phase-coded pulses. Alternatively, a group of short pulses can be transmitted with short gaps between the pulses. Different techniques for measuring the severely overspread targets based on these ideas have been presented by several authors (e.g. Farley, 1969, 1972; Sulzer, 1986a,b; Lehtinen and Häggström, 1987; Sulzer, 1993).

An opposite to the severely overspread targets are the underspread ones. Their scattering spectrum is much narrower than the PRF and they can be sufficiently sampled with a lag resolution lower than the signal roundtrip time to and from the furthermost target range. Thus, the underspread targets can be measured with uniformly spaced pulses, in the simplest situation with a pulse length corresponding the desired range resolution.

Between the two extreme situations are the moderately overspread targets. They require too high a lag resolution to be considered as underspread ones, but at least some parts of the target require lag values longer than is possible to measure with the long pulses. In other words, the spectrum of the target is only slightly wider than the maximum PRF. This kind of target cannot be properly measured with an experiment using a constant pulse-repetition frequency.

Published by Copernicus Publications on behalf of the European Geosciences Union. 
A novel method for measuring moderately overspread radar targets has been presented by Uppala and Sahr (1994). The method, called Aperiodic Transmitter Coding (ATC), is based on transmitting the radar pulses in an aperiodic manner, which enables the estimation of the pulse-to-pulse correlations with a lag resolution higher than the target range extent. The method has already been proven to be advantageous when studying the equatorial spread $\mathrm{F}$ (Hysell and Chau, 2004; Chau et al., 2004; Hysell et al., 2008a). The same method is in use with weather radars, where it is used as a solution for the so-called range-Doppler dilemma and is known under the name Simultaneous Multiple Pulse-Repetition Frequency (SMPRF) (Pirttilä et al., 2005). Furthermore, aperiodic pulsing has been used for a long time in the so-called "Staggered PRF" method of Moving Target Identification (MTI) radars (e.g. Skolnik, 1990; Schleher, 1991), but with considerably different data analysis due to a totally different kind of a target than presented in this paper.

In the altitude interval from $50 \mathrm{~km}$ to $1000 \mathrm{~km}$, which is roughly the interval routinely detectable with the EISCAT incoherent scatter radar facilities, the correlation time of the scattered signal has large variations. In the D-region altitudes, the signal correlates over several milliseconds, whereas the first zero of an F-region ACF is typically around one hundred microseconds when measured with the VHF radar. The time taken for an electromagnetic pulse to travel from the ground level to the $50 \mathrm{~km}$ range and back is less than half a millisecond, which is much less than the expected correlation time at the D-region altitudes. Thus, it is not possible to measure the D-region with an experiment consisting of pulses longer than the correlation time of the scattered signal.

If one is only interested in lag values longer than the longest correlation times in the F-region, the D-region can be considered as an underspread target and measured with an experiment consisting of pulses transmitted with short uniform time intervals. With this kind of arrangements, a sufficient lag resolution of about one millisecond can be easily achieved. However, the experimenter is usually also interested in measuring the short lags to obtain reliable estimates of the actual backscatter power. Thus, it would be desirable to combine the aperiodic transmitter coding and the long phase-coded pulses in a way allowing the estimation of both the short F-region correlations and the long D-region correlations from the same data.

In a preliminary paper (Virtanen et al., 2008b), incoherent scatter radar experiments with phase-coded pulses transmitted in an aperiodic manner were introduced. The phase coding allowed accurate measurements of the short intra-pulse lags, which are necessary for the E- and F-regions of the ionosphere. By using several different inter-pulse periods, it was possible to cover a continuous range interval larger than the longest IPP, but still measure also the pulse-to-pulse correlations needed for the D-region analysis with a sufficient lag resolution. The experiments were successful in the sense that it was possible to analyse the data with lag profile inversion (Virtanen et al., 2008a; Damtie et al., 2002), but the actual estimation accuracies of the experiments were not evaluated at all. The IPPs were also chosen in a rather arbitrary way, leading to large differences in the experiment efficiencies at different ranges.

In this paper, experiments combining the simple difference cover codes presented in Uppala and Sahr (1994) and long phase-coded pulses are introduced and evaluated. Some other ATC codes are better in a certain sense and published in both Uppala and Sahr (1994) and in Uppala and Sahr (1996), but we are willing to use the simple difference cover codes to be able to fill the large duty cycle $(12.5 \%)$ of the EISCAT mainland radars. For a more detailed discussion of simple difference covers, see Clinger and Van Ness (1976). The simple difference cover codes also help us to get a uniform lag resolution for the pulse-to-pulse lags and to keep the experiment efficiency about equal at all ranges. We will adopt the name Phase-coded Pulse Aperiodic Transmitter Coding (PPATC) for the new experiments to underline the differences between the basic ATC experiments without phase coding and the method of alternating the phases of the whole pulses as proposed by Chau et al. (2004). The PPATC method is also different from the combination of alternating codes and multipulse codes (Nygrén et al., 1996), because our aim is to estimate all lag values, including the intra-pulse lags shorter than the pulse length and arbitrarily long pulseto-pulse lags. The continuous cycle of the ATC coding, with several pulses inside the target at all instances of time, is also considerably different from the separated pulse groups of the multipulse codes used in Nygrén et al. (1996).

We will demonstrate how ATC and phase-coded pulses may be combined to make the experiment approximately equally efficient at all target ranges. The problem of selecting phase codes suitable for the PPATC experiments is also shortly discussed. Finally, a PPATC experiment is evaluated and compared to other experiments for both low and high SNR using lag profile inversion and simulated data. A test run of the experiment has also been performed with the EISCAT VHF (224 MHz) radar. A sample ACF from this experiment is shown at the end of the paper.

\section{Filling the radar duty cycle}

A simple design of a radar experiment is to transmit pulses of duration $t_{p}$ with an inter-pulse period $t_{i}$ between the pulses. After each transmitted pulse, the echoes from the pulse are recorded until the start of next pulse. If the target range extent is $r_{t}$, one chooses $t_{i}=2 r_{t} / c$, where $c$ is the speed of light. Then, it is straightforward to select the pulse length $t_{p}$ in a way leading to the maximum allowed duty cycle of the radar.

In ionospheric conditions typical for the EISCAT mainland radars, there are two major drawbacks in the described method: 
Table 1. Relative lengths of the $\mathrm{N}$ inter-pulse periods of simple difference covers of length $N$.

\begin{tabular}{|c|c|}
\hline$N$ & relative IPPs \\
\hline 2 & $1: 2$ \\
\hline 3 & $1: 2: 4$ \\
\hline 4 & $\begin{array}{l}1: 2: 6: 4 \\
1: 3: 2: 7\end{array}$ \\
\hline 5 & $1: 3: 10: 2: 5$ \\
\hline 6 & $\begin{array}{l}1: 7: 3: 2: 4: 14 \\
1: 3: 6: 2: 5: 14 \\
1: 3: 2: 7: 8: 10 \\
1: 2: 5: 4: 6: 13 \\
1: 2: 7: 4: 12: 5\end{array}$ \\
\hline 8 & $\begin{array}{c}1: 4: 22: 7: 3: 6: 2: 12 \\
1: 6: 12: 4: 21: 3: 2: 8 \\
1: 4: 2: 10: 18: 3: 11: 8 \\
1: 3: 5: 11: 2: 12: 17: 6 \\
1: 3: 8: 2: 16: 7: 15: 5 \\
1: 2: 10: 19: 4: 7: 9: 5\end{array}$ \\
\hline 9 & $\begin{array}{l}1: 4: 7: 6: 3: 28: 2: 8: 14 \\
1: 11: 8: 6: 4: 3: 2: 22: 16 \\
1: 6: 4: 24: 13: 3: 2: 12: 8 \\
1: 2: 4: 8: 16: 5: 18: 9: 10\end{array}$ \\
\hline
\end{tabular}

1. The high radar duty cycle and target range extent of several hundred kilometers would lead to large $t_{p}$, i.e. long transmission pulses. For this reason, the leading edge of the pulse has already passed the lower ionosphere at the time when signal reception is started. Thus, the amount of echo samples with contribution from each range - the radar efficiency - is low in the D- and E-regions with respect to the high efficiency in the F-region.

2. The inter-pulse period $t_{i}$, when calculated in this way, is usually larger than sufficient for the D-region pulse-topulse correlation measurements.

Due to the problems mentioned, the EISCAT experiments are usually roughly divided into F-region, E-region and D-region experiment groups. The F-region experiments are based on long pulses with long IPPs, and therefore rather inefficient in the D- and E-regions. In contrast, the D- and E-region experiments are using shorter pulses and shorter IPPs, assuming that the echoes from above the one-IPP range will either be insignificant or will have noise-like behaviour in the decoding process. The assumptions may be well justified in most cases but, if not carefully taken into account in experiment design, the F-region echoes still have the capability of biasing the decoded lag profiles.

A well-known and very beneficial way to avoid problems with unwanted echoes is to divide the transmissions into sev-

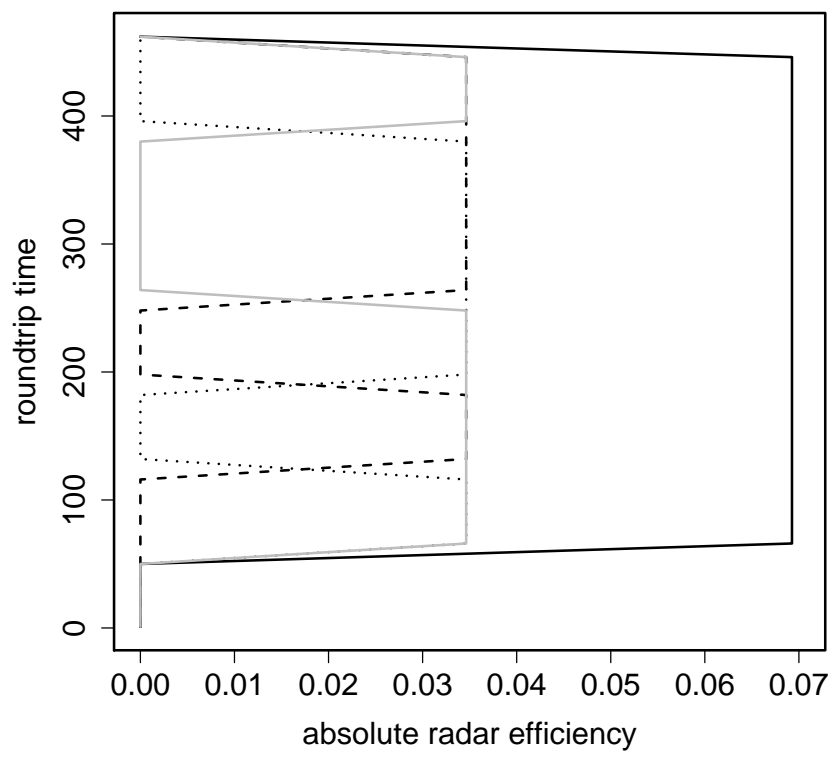

Fig. 1. The absolute radar efficiency of zero-lag of a PPATC experiment with a pulse length of 16 units and three different IPPs (66, 132 and 264 units). The dashed, dotted and grey lines are the efficiencies of the three individual pulses. The solid black line is the total efficiency of the experiment. A time period of 50 units, in addition to the pulse length, is assumed to be lost during each transmission.

eral frequency channels. This method definitely solves the problem in the cases of the severely overspread E- and Fregions but, simultaneously, removes the possibility of pulseto-pulse correlation measurements in the moderately overspread D-region, because the signals at different frequencies do not correlate. Recently, Raizada et al. (2008) have also used a method of subtracting the F-region contribution, measured with a different experiment, from D-region IS spectra.

In Virtanen et al. (2008b), we demonstrated the idea of transmitting rather short pulses in the same frequency with non-uniform IPPs. Data from such experiments was possible to analyse, but the experiment efficiency, the number of independent lagged products collected, had large variations as a function of range. The pulse-to-pulse lags provided by the experiments were also rather arbitrarily spread over a large number of lag values, making the data unnecessarily complicated to use.

Both problems mentioned can be avoided, if the IPPs are selected using the simple difference cover codes of Uppala and Sahr (1994). These codes are continuous cycles of $N$ pulse separations, selected to include all pulse separations from 1 to $N(N-1)+1$ exactly once per cycle. A complete list of all simple difference covers of lengths $N=2 \ldots 9$ is given in Table 1. Instead of the original notation of Clinger and Van Ness (1976) and Uppala and Sahr (1994), which is based on the pulse positions, we are using the one based on 
the pulse separations (IPPs), because it then follows more closely the notation used in Virtanen et al. (2008b).

When the IPP cycle of a simple difference cover is continuously repeated, all integer-valued pulse separations from unity to arbitrarily long values are produced. When calculating lagged products for autocorrelation function estimation, the co-located pulses producing correlating echoes are separated by $N(N-1)+1$ units in roundtrip time. The original idea behind ATC is to select large enough $N$ to guarantee that only the lowest co-located pulse pair is inside the detectable target. In this paper, the range corresponding $N(N-1)+1$ units in roundtrip time is called the "range coverage", as it is both the first "blind" range of the experiment and usually also the range below which the whole detectable target is assumed to be located.

In PPATC the simple difference covers are used as follows. Let us mark the time unit of the ATC coding by $T$ and the length of the ATC code (the number of different IPPs) by $N$. Now, if $T$ is larger than or equal to the time period lost while transmitting a pulse, the echoes from at least $N-1$ pulses out of the $N$ possible ones are collected at each range from $T$ to $(N(N-1)+1) T$. This is demonstrated for $N=3$ in Fig. 1, where the smallest possible $T$ is chosen, leading to a constant radar efficiency as a function of range. Due to hardware limitations and ground clutter, echoes are lost from a much longer period of time than the actual pulse length during each transmission.

The simple difference covers also guarantee that the pulseto-pulse lags are evenly distributed in the lag direction. At each range, at least $N-1$ pulse-to-pulse lags out of the $N(N-1)$ possible ones can be measured from the interval $[T, N(N-1) T]$, but the lag values are not the same at all ranges. In addition, at each pulse-to-pulse lag from $T$ to $N(N-1) T$, only one of the $N$ possible pulse pairs is colocated. Thus the radar efficiency of these lags is smaller than the efficiency of the short intra-pulse lags. At the lag values $k(N(N-1)+1) T, k=1,2,3, \ldots$, the lag profiles are continuous and all ranges are again covered with at least $N-1$ pulse pairs. An example of the radar efficiencies of both the intrapulse and pulse-to-pulse lags for a PPATC experiment with 3 different IPPs can be found in the first panel of Fig. 2. In practical experiment design, the time unit $T$ can be chosen to be large enough to allow the estimation of all possible pulseto-pulse lags in the D-region ranges.

From a geophysicist's point-of-view, covering large continuous range intervals with a single experiment would be advantageous. For this reason, the present paper concentrates in designing an experiment with pulses long enough for a reasonable E- and F-region analysis and an ATC coding allowing high enough a lag resolution for the D-region. Experiments mainly dedicated to the D-region, combining short (around $100 \mu \mathrm{s}$ ) pulses and a range coverage of about $400 \mathrm{~km}$, can be easily designed with the same method. The latter kind of experiments are important in D-region studies, because they allow the estimation of the short lags without running into problems with the F-region echoes, and also improve the lag resolution of the pulse-to-pulse lags, when compared with an experiment with the same pulse length and uniform IPPs.

\section{Experiment radar efficiencies}

In this section, the absolute radar efficiencies of different IS radar experiments are compared. By the term "absolute radar efficiency" we mean the fraction of the experiment total duration during which lagged products from a given range at a given lag value can be collected. The experiments are designed to have the same duty cycle and the same bit length $(15 \mu \mathrm{s})$ in phase coding. An optimised code sequence (see Sect. 4) is used in the PPATC experiment. Other experiments used in the comparisons are based on strong type 1 alternating codes (Lehtinen and Häggström, 1987; Markkanen and Nygrén, 1997; Nygrén and Markkanen, 1997).

To include the limitations due to the radar hardware and ground clutter, we assume $90 \mu$ s of echoes to be lost before each transmitted pulse, due to the limitations of the radar, and $330 \mu$ s of echoes to be lost after each pulse, due to ground clutter. Furthermore, we assume that the transmitter must be turned on $40 \mu$ s before the start of an actual pulse, as is the case with the EISCAT mainland radars. The last requirement makes the maximum RF duty cycle dependent on the pulse length.

The PPATC experiment used in the comparison has a pulse length of $240 \mu$ s and the IPPs are 990, 1980 and $3960 \mu$ s. Thus, the experiment range coverage as defined in Sect. 2 is $(990+1980+3960) \mu \mathrm{s} \times 0.15 \mathrm{~km} / \mu \mathrm{s} \approx 1000 \mathrm{~km}$. All intrapulse lags from 0 to $240 \mu$ s can be inverted from the experiment. In addition, pulse-to-pulse correlations can be measured with a lag resolution of $990 \mu \mathrm{s}$, as seen in the first panel of Fig. 2. The time unit $T$ of the ATC coding is selected to be large enough to allow the estimation of all pulse-to-pulse lags at the D-region altitudes. An example of raw voltage data recorded from a test run of the PPATC experiment is plotted in Fig. 3.

The PPATC experiment is compared to three uniform-IPP experiments, all of which have approximately the same duty cycle as the PPATC experiment. The first one (exp1) has the same continuous range coverage as the PPATC experiment. To fulfill the range coverage requirement, long pulses and long IPPs need to be used. This makes the experiment very inefficient in the D-region and practically prevents reasonable pulse-to-pulse correlation measurements, which would typically require a lag resolution of the order of $1 \mathrm{~ms}$ (second panel of Fig. 2).

The second uniform-IPP experiment (exp2) uses the same pulse length as the PPATC experiment. When used with uniform IPPs, the range coverage is significantly reduced from that of the PPATC experiment. However, the pulses are closer than in exp1, possibly allowing pulse-to-pulse measurements 

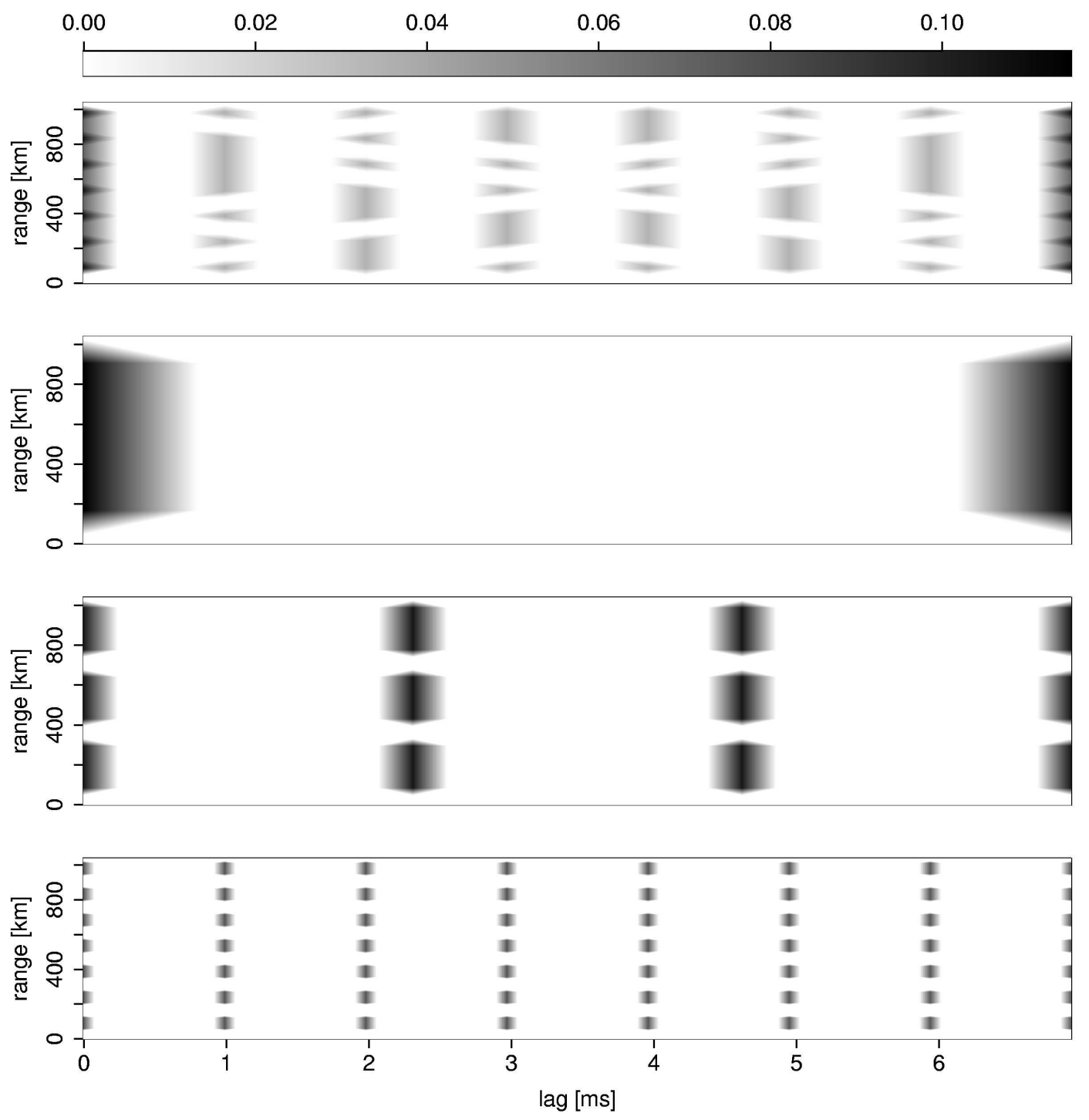

Fig. 2. Absolute radar efficiencies as functions of range and lag for the experiments compared in this paper. Following the names in Table 2, the efficiency of the PPATC experiment is plotted in the top panel, exp1 in the upper middle panel, exp2 in the lower middle panel and exp3 in the bottom panel.

of the longest correlations of the D-region (third panel of Fig. 2).

The third uniform-IPP experiment (exp3) has the same lag resolution with the PPATC experiment for the pulse-to-pulse lags. In other words, the IPPs are very short, leading to a very small range coverage. In addition, the pulses need to be short, reducing the actual RF duty cycle due to the technical limitations of the klystron radar. The absolute radar efficiency of exp3 is plotted in the lowest panel of Fig. 2. The pulse lengths, IPPs, range coverages and RF duty cycles of the experiments are listed in Table 2.

From Fig. 2 it is evident that the PPATC experiment combines the large coverage of exp1, the pulse length of exp2 and the short IPPs of exp3. This combination is achieved by sacrificing part of the radar efficiency of the short lags in the F-region and part of the lag coverage of exp1.

\section{Experiment evaluation}

The evaluation in the previous section only revealed that there is a possibility to collect certain amounts of lagged products with contributions from certain lag and range values. However, the samples contain range ambiguities due to the long pulses and, in the case of the PPATC experiment, because several pulses are simultaneously inside the target. To show that the new experiment really is beneficial, we have 

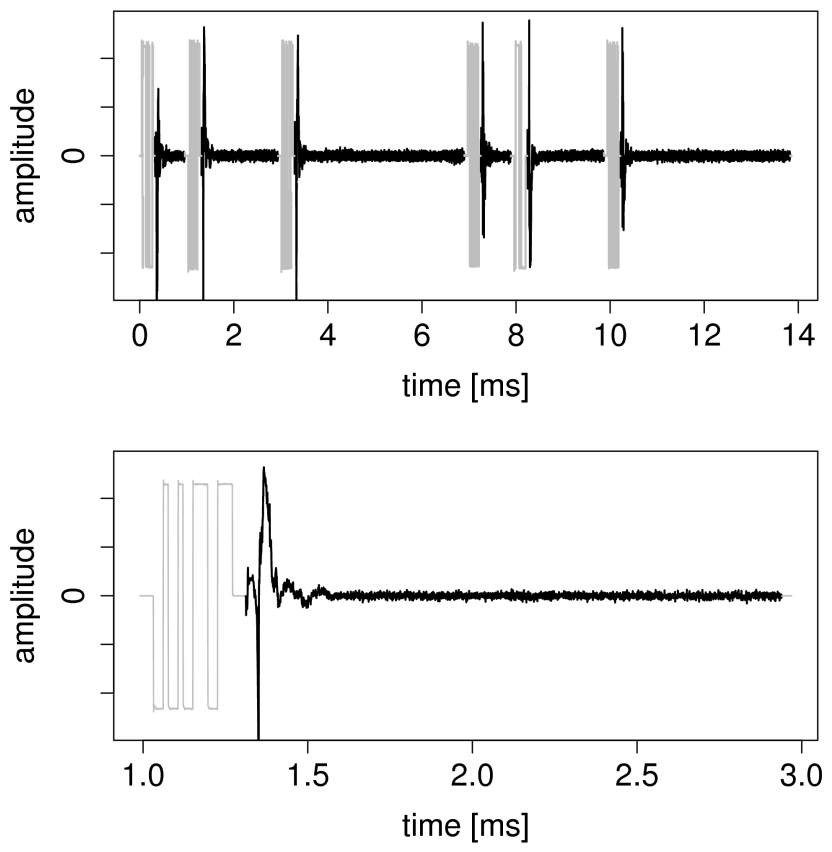

Fig. 3. The real part of raw voltage data from the PPATC experiment. The data is from an experiment performed with the EISCAT Troms $\emptyset$ VHF radar on 28 November 2008. Upper panel: Transmission signal (grey) and the echoes (black) of two full cycles of the ATC coding. Lower panel: The second transmission pulse of the upper panel and the subsequent echoes. A strong ground clutter signal is seen immediately after the pulse. When analysed without clutter suppression, the useful incoherent scatter signal begins around $1.6 \mathrm{~ms}$ in the plot.

to confirm that we are able to remove the range ambiguities from the data accurately.

As proposed in Virtanen et al. (2008b), the PPATC experiments can be evaluated using lag profile inversion (Virtanen et al., 2008a). In lag profile inversion, the conventional decoding filter based methods for removing range-ambiguities from ACF estimates are replaced by a statistical inversion based method. A range-profile of the ACF at a fixed time-lag is called a lag profile. The true lag values at each range-gate are unknowns and, using measured transmission envelopes, a set of linear equations relating the measured ambiguous lagged products to the unknown true lag values is produced. Statistical inverse methods (Orispää and Lehtinen, 2009) are then used to estimate the true lag profile. The procedure is repeated to all lag values necessary to properly sample the whole ACF.

In the experiment evaluation, fictitious measurements with the given phase codes and IPPs are created and the data is analysed with a unit variance given to the ambiguous lagged products. As described in Virtanen et al. (2008a), the inversion provides the full posteriori covariance matrix, from which only the variances are used in this evaluation. No addi-
Table 2. The experiments used in the comparisons. The listed experiments have approximately the same "beam duty cycle" $(12 \%)$, the differences in the RF duty are mainly due to the requirement of starting the transmitter $40 \mu \mathrm{s}$ before the actual pulse. All of the experiments use the same bit length $(15 \mu \mathrm{s})$ in phase-coding. An optimised code sequence is used in the PPATC experiment and a strong type 1 alternating code in the other experiments. exp1 has the same range coverage, $\exp 2$ the same pulse length and exp3 the same pulse-to-pulse lag resolution as the PPATC experiment.

\begin{tabular}{ccccc}
\hline & $\begin{array}{c}\text { pulse length } \\
{[\mu \mathrm{s}]}\end{array}$ & $\begin{array}{c}\text { IPP } \\
{[\mu \mathrm{s}]}\end{array}$ & $\begin{array}{c}\text { max range } \\
{[\mathrm{km}]}\end{array}$ & $\begin{array}{c}\text { RF duty } \\
{[\%]}\end{array}$ \\
\hline PPATC & 240 & $990 ; 1980 ; 3960$ & 1040 & 10.4 \\
exp1 & 795 & 6930 & 1040 & 11.5 \\
exp2 & 240 & 2310 & 347 & 10.4 \\
exp3 & 75 & 990 & 149 & 7.6 \\
\hline
\end{tabular}

tional information, i.e. regularisation, is included in the inversion process. The same range resolution, the phase-coding bit length, is used at all ranges. The posteriori variances achieved this way are equal to the actual variances of the inverted lag profiles, if the signal-to-noise ratio of the received signal is infinitely low. In addition to this evaluation, a case study of the experiment behaviour for high-SNR is included in Sect. 5.

In the low-SNR evaluation, the variances presented in this paper are scaled very much in the same way as in Lehtinen et al. (2008), where a unit variance was given to an experiment with perfect decoding properties. To allow a comparison between any two experiments with the same bit length in the phase coding, we are this time scaling the variances by the total duration of the full transmission cycle of the experiment. In this scaling, an experiment with a perfect phase coding and $100 \%$ duty cycle in both transmission and reception would have unit variance. For real experiments with a smaller duty cycle, the minimum scaled variance is equal to the inverse of the absolute radar efficiency at the given lag value.

In the actual code optimisation, the theory matrix $\mathbf{A}$, as described in Virtanen et al. (2008a), was slightly modified to normalise the optimum variance to unity in all range gates, regardless of the actual radar efficiency at the gate. This normalisation allows us to perform the code optimisation by minimising the sum of variances, i.e. the trace of the posteriori covariance matrix, using the algorithm of Vierinen et al. (2008). The smallest possible IPPs were also used to keep the inverse problem as small as possible. The variances presented in this paper are normalised in a different way, because the authors find it more convenient to present the actual variances, including the effects of radar efficiency. The evaluation is also performed with the IPPs of the actual experiments, not the smallest possible ones used in the code search. 
Lag 1

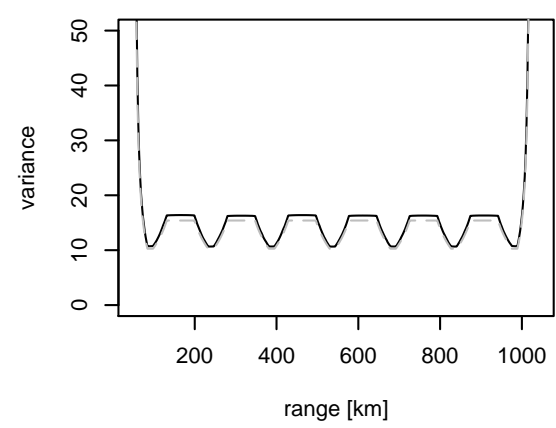

Lag 6

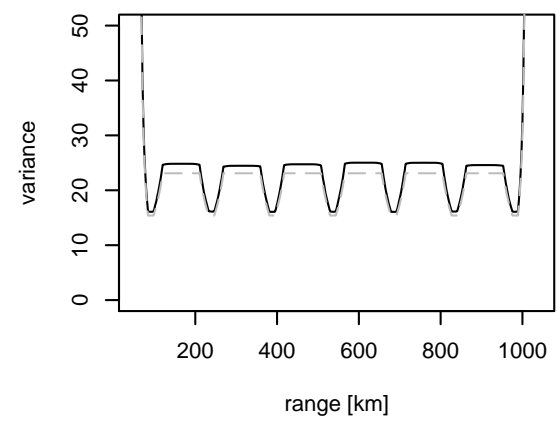

Lag 11

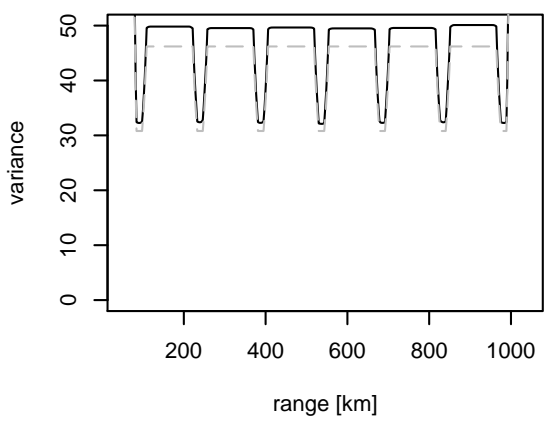

Fig. 4. Normalised variances of selected lags of the PPATC experiment with 48 phase-coded pulses and 3 different IPPs. The continuous black line is the actual posteriori variance of the lag profile and the grey dashed line is the smallest possible variance with the given IPPs and pulse lengths.

The evaluation process revealed that the PPATC experiments require much longer code sequences than the standard experiments, which are decoded only up to the one-IPP range. For the experiment with 3 different IPPs, a sequence of 48 phase codes was selected for further evaluation. When analysed with the range and lag resolutions equal to the bit lengths of the codes, the average lag profile variances are found to be $6.8 \%$ larger than the theoretical minimum values. The evaluated variances and the theoretical optimal values of selected lag profiles are plotted in Fig. 4.

Normalised variances of selected lag profiles of the four experiments are plotted in the first two panels of Fig. 5. In the top panel, the experiments are analysed only up to the oneIPP range. In this case, the variances of exp1, exp2 and exp3 are equal to the theoretical minimum with the given pulse lengths and IPPs, because they are based on strong type- 1 alternating codes, which are known to have perfect decoding properties. It is possible to extend the analysis of all four experiments to the same maximum range but, in that case, the experiments with uniform IPPs will have larger variances, as seen in the middle panel of Fig. 5. This is because the alternating codes are not designed for decoding above the one-IPP range. The problems with the large variances could be avoided by modifying the alternating codes in a way allowing perfect decoding above the one-IPP range. A method for generating this kind of codes is known, but the modified code sequences are longer than the standard type 1 alternating codes. A more severe problem are the data gaps in the range direction, which cannot be avoided with the uniform IPPs (see the Figs. 2 and 5).

The long pulses of exp1 make it the most accurate in the F-region, while it has rather poor variances in the lowest ranges. A more detailed plot of the bottom part variances is found in the top panel of Fig. 6. At the first lag, the PPATC experiment has variances somewhat comparable to those of exp1 also in the largest ranges but, with increasing lag num- bers, the long pulses of exp1 make it superior when compared with the other experiments. This analysis, as well as the variances presented in Fig. 4, reveal that it is possible to analyse the PPATC experiment with lag profile inversion over the whole large range interval without using any kind of regularisation. However, the pulse length should be carefully selected to keep the radar efficiency of the longest required intra-pulse lags at an acceptable level.

In the case of the pulse-to-pulse correlations, we assume that the signal from above $1 \mathrm{~ms}(150 \mathrm{~km})$ does not correlate. We will limit our comparison to the two experiments with the best lag resolutions: the PPATC experiment and the uniformIPP experiment with the shortest pulses (exp3). A remarkable property of the PPATC experiment is that the same lag resolution is achieved for the pulse-to-pulse lags with these two experiments, though the pulse length of the PPATC experiment is three times that of exp3 and both have the largest possible duty cycles. Simultaneously, the PPATC experiment has a range coverage seven times that of exp3 and its long pulses allow several intra-pulse lags to be measured from the severely overspread F-region.

Already the radar efficiencies in Fig. 2 revealed that the uniform-IPP version is more efficient at certain lag values, but the PPATC version provides much more separate nearby lag profiles. To make a somewhat fair comparison, we will compare the "full" pulse-to-pulse correlations $(990 ; 1980 ; \ldots$ $\mu \mathrm{s})$ with all possible fractional lags included in each "full" lag. Thus, the first pulse-to-pulse lag of the PPATC experiment is an average of the $750-1230 \mu$ s lag profiles and the equivalent lag of exp3 is an average of the $915-1065 \mu$ s lag profiles. Because the averaging increases the number of independent lagged products, the normalised variances of these lags are much better than those of the intra-pulse lags. The integration in lag is usually acceptable in the D-region due to its narrow scattering spectra. If the integration is not acceptable, the corresponding information can still be extracted 
Lag 1
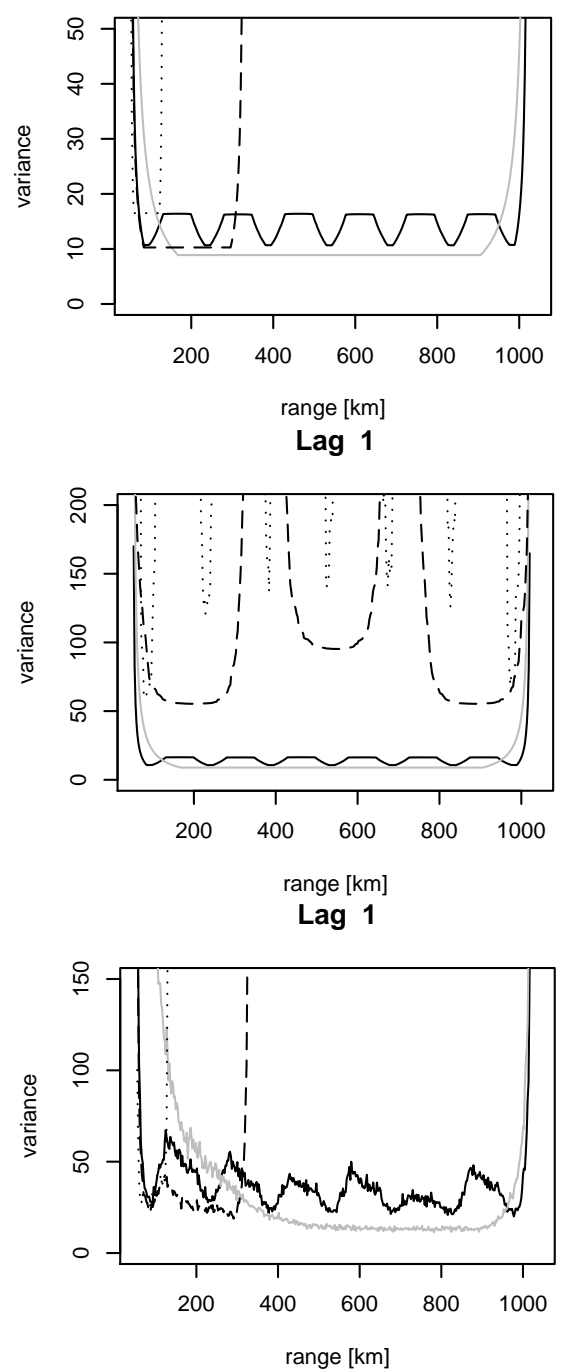

Lag 6
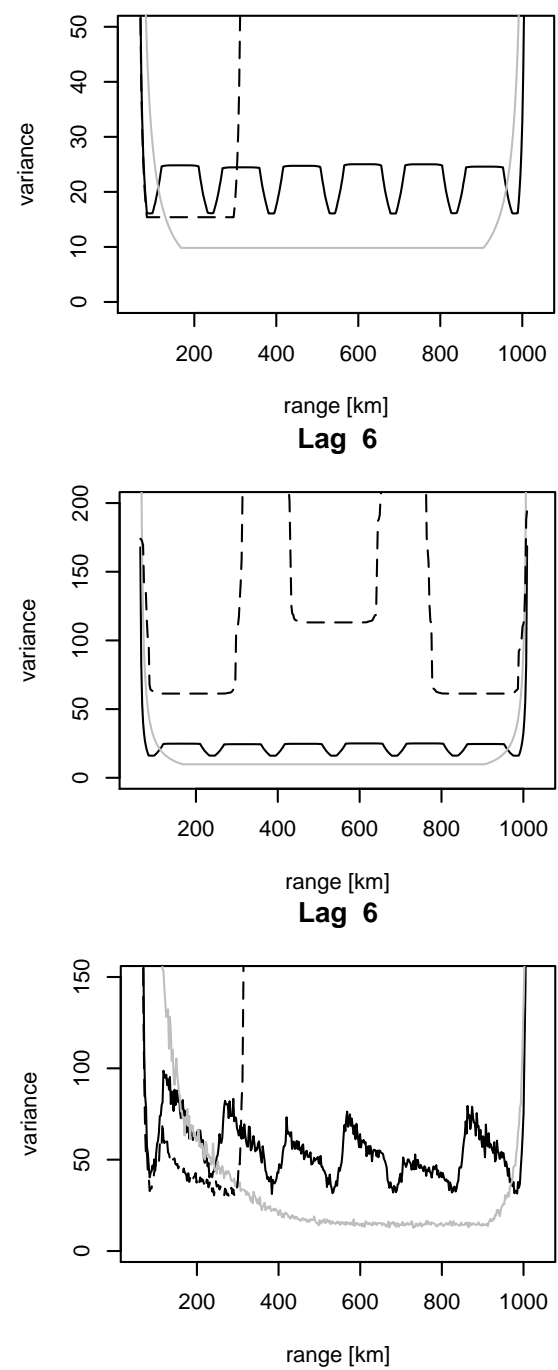

Lag 11
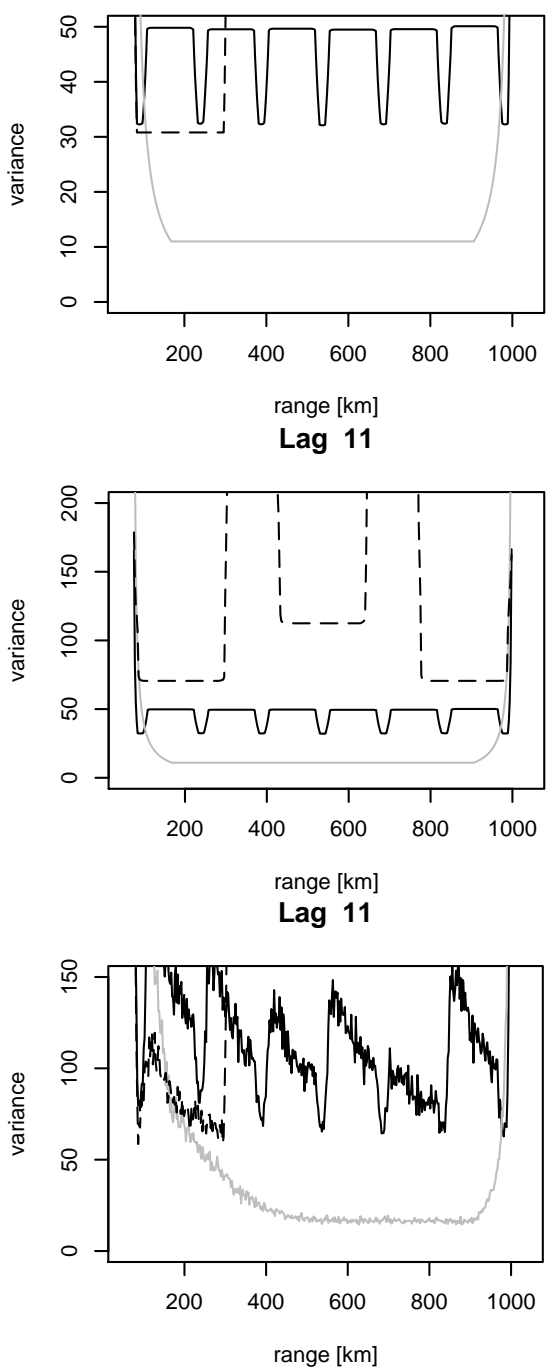

Fig. 5. Top panel: Normalised variances of selected lags under low SNR conditions in the compared experiments. The continuous black line is the variance of the PPATC experiment, the grey line is the variance of exp1, the dashed line the variance of exp2 and the dotted line is the variance of exp3. The experiments with uniform IPPs are analysed only up to the range of one IPP. Middle panel: Normalised variances of the compared experiments under low SNR conditions, when the analysis of all four experiments is extended to cover the target range interval of the PPATC experiment. It is clear that the strong type-1 alternating codes used in the experiments do not behave well when analysed this way. Bottom panel: sample variances of the inversion results of simulated data. In total 314 inversion results have been used for each variance estimate.

from the data by calculating separate lag profiles for all fractional lag values.

From the middle panel of the Fig. 6, we can see that both experiments are rather good in the D-region for low SNR. As expected, the uniform-IPP version is more efficient in the very lowest range gates, as it has shorter pulses and a higher pulse-repetition frequency on average. However, the PPATC experiment is equally good with exp3 already at the $75 \mathrm{~km}$ range and, due to its higher RF duty cycle, is even slightly better in the range interval from 80 to $100 \mathrm{~km}$.

\section{Analysis of simulated data}

In order to make a case study of the experiment behaviour under more realistic SNR conditions, simulated radar echoes were generated and analysed for all four experiments. The scattering spectra as functions of height were based on plasma parameters calculated with the IRI2007 model. The parameters were calculated for the geographical coordinates of the EISCAT Troms $\varnothing$ radar $\left(69^{\circ} 35^{\prime} 11^{\prime \prime} \mathrm{N}, 19^{\circ} 13^{\prime} 3^{\prime \prime} \mathrm{E}\right)$ on 1 July 2007 at 11:00 UT. The simulation produced "clean" 
Lag 1
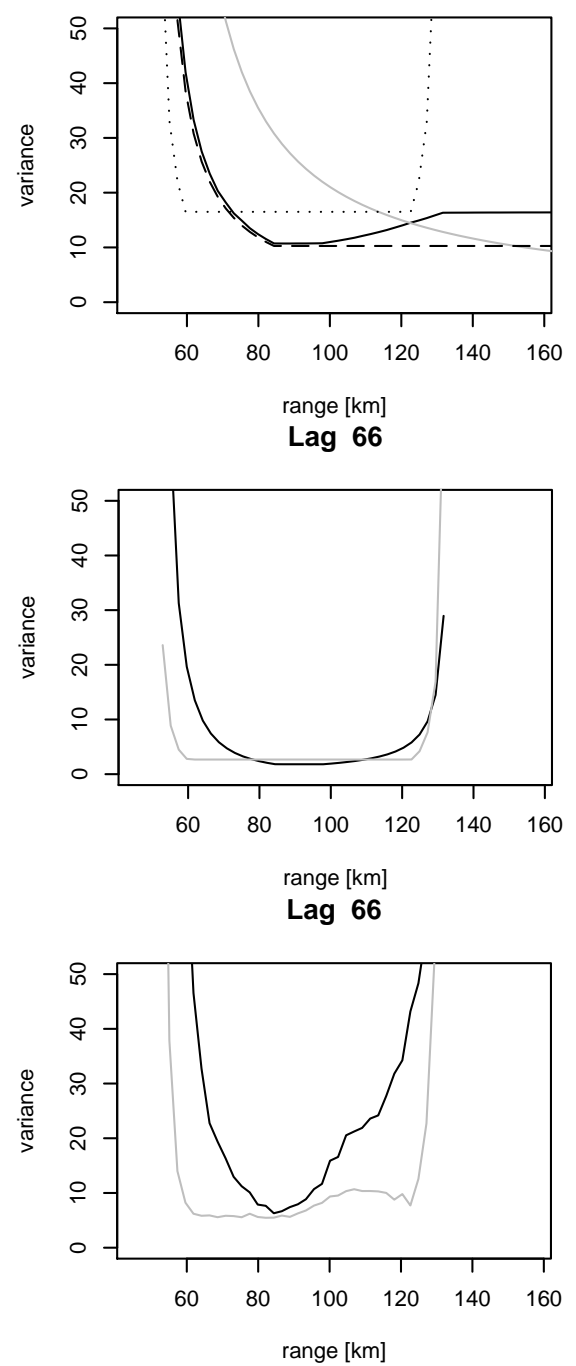

Lag 6
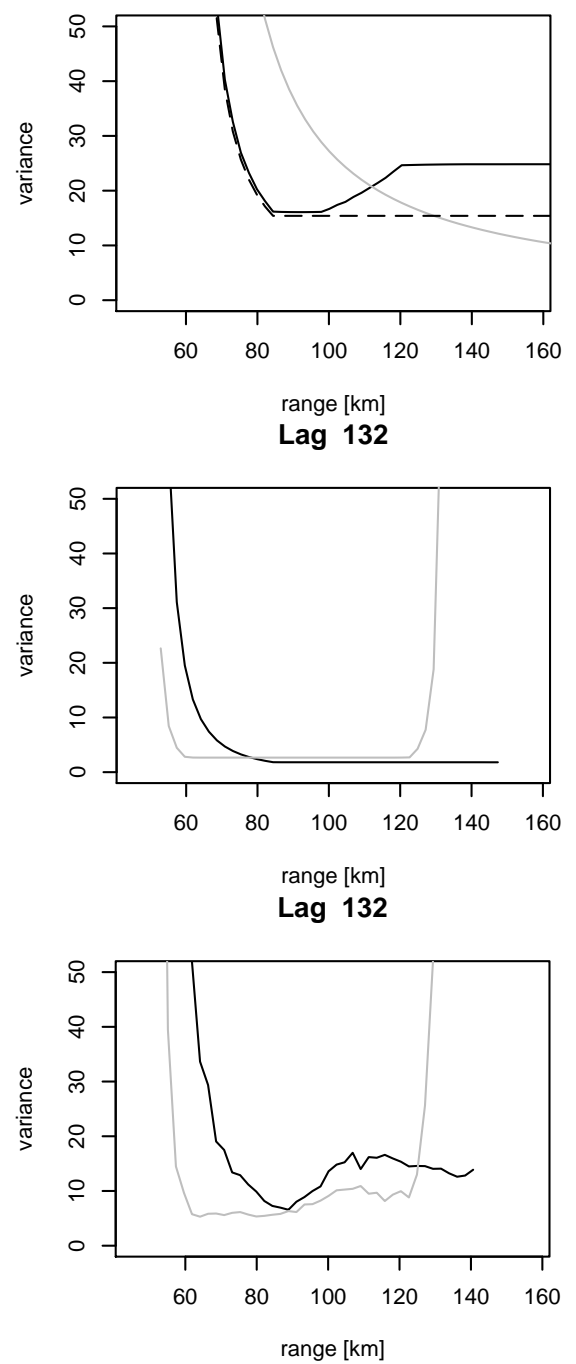

Lag 11
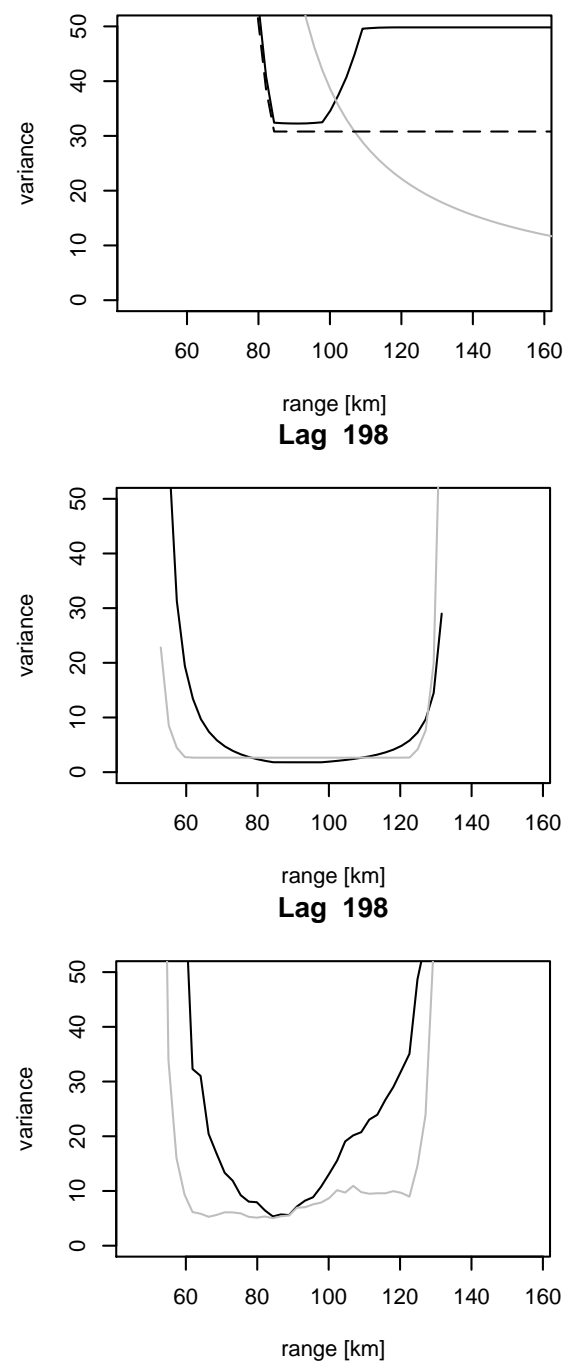

Fig. 6. Top panel: The bottom parts of the normalised lag profile variances in the compared experiments. The black, grey, dashed and dotted lines are variances in PPATC experiment, exp1, exp2 and exp3, respectively. The differences in the estimation accuracies at the lowest ranges are clearly visible. Middle panel: Normalised variances for the pulse-to-pulse lags of the PPATC experiment and exp3 for low SNR. All possible fractional lags around each main pulse-to-pulse lag are included in the same profiles. The lags are numbered as multiples of the bit length of the phase codes. Bottom panel: sample variances of the inversion results of simulated data for the pulse-to-pulse lags. In total 314 inversion results have been used for each variance estimate.

radar echoes with the given spectra. After the simulation, normally distributed random noise was added to the echoes. The simulation was performed with a $5 \mu$ sampling interval, i.e. triple oversampling when compared to the bit length of the phase-coding. The power of the added noise was adjusted to approximately equal the maximum power of the simulated "ionospheric" signal of exp1. In the analysis, the data was filtered using a low-pass filter, which still increases the signal-to-noise ratio. Thus, the evaluation is performed with data corresponding to good signal-to-noise conditions, making the self-noise effects clearly visible in the results.
For this evaluation, the code sequence of the PPATC experiment was made "strong" by repeating the code sequence twice and multiplying every second bit of the latter half by -1 . Thus, the total length of the code sequence has grown to 96 codes. The method, introduced e.g. in Sulzer (1989), maintains the code properties in uniform-IPP experiments when the data is filtered using a boxcar filter with an impulse response equal to the bit length of the code. In PPATC experiments, several pulses are simultaneously inside the target ranges. In this situation, the effect of the procedure is slightly different, but the properties of the modified code sequence 


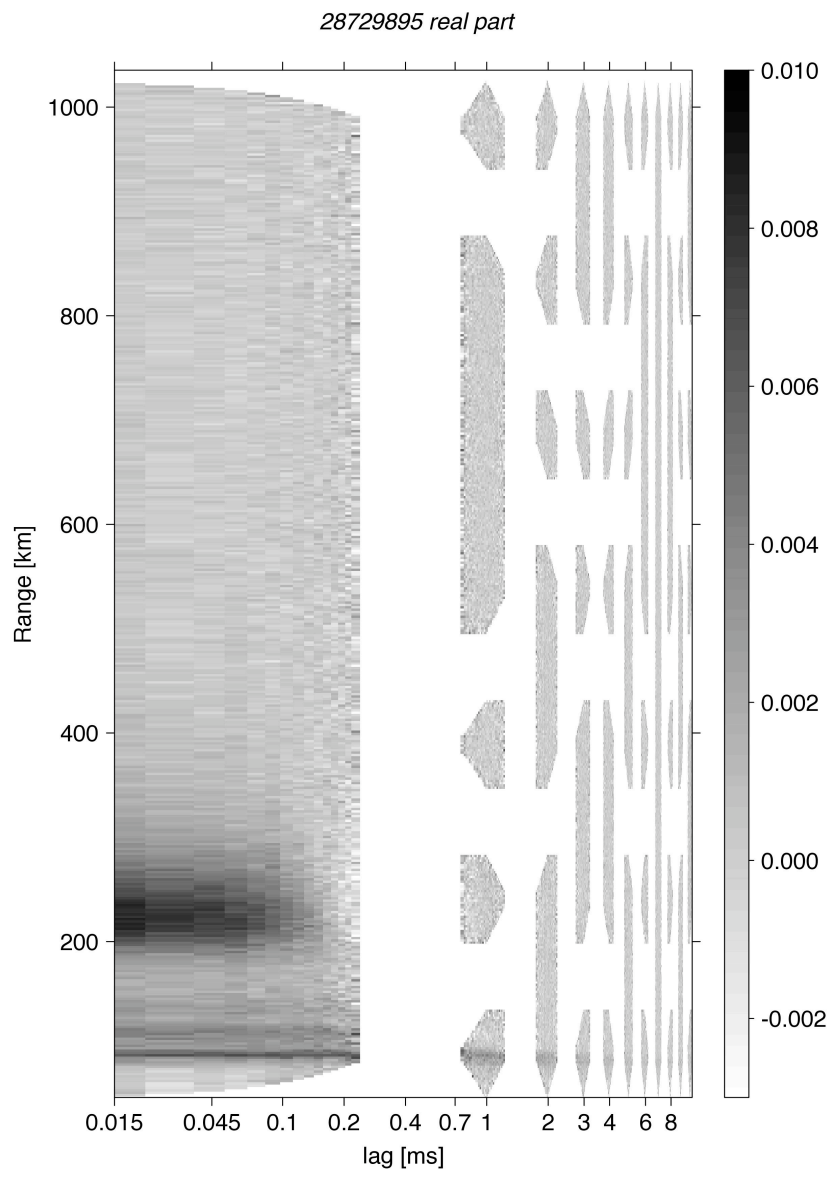

Fig. 7. The real part of the plasma scattering autocorrelation function measured with the EISCAT Troms $\varnothing$ VHF radar on 28 November 2008 (in arbitrary units). The ranges from 50 to $1000 \mathrm{~km}$ are continuously covered with the short ( 15 to $225 \mu$ s) lag profiles. In addition, several pulse-to-pulse lag profiles with a lag resolution of $990 \mu$ s are measured. To underline the possibility of measuring the pulse-to-pulse correlations from all ranges, the E- and F-regions are also contained in the pulse-to-pulse part. Neither are nearby pulseto-pulse lags integrated in the same profiles to demonstrate that the whole lag coverage presented in Fig. 2 is really possible to achieve.

are still very close to the original one, except that the new one is expected to be much more filter-tolerant.

In the analysis, a time resolution of $0.88704 \mathrm{~s}$ was used. During this period of time, the full transmission cycle of the PPATC experiment is repeated exactly four times and the cycles of exp1, exp2, and exp3 are repeated once, 12 times and 56 times, respectively. Thus, none of the experiments will suffer from incomplete code sequences being included in the analysis. In total 314 integration periods were analysed for all four experiments and the mean values and the sample variances of the resulting ACFs were calculated for each experiment separately. In this paper, only a qualitative comparison of the lag profile variances is included. A more detailed eval- uation of the covariances between different range gates and lags, as well as code optimisation to minimise these covariances, may be the subject of a future study.

The sample variances of the inverted lag profiles are plotted in the bottom panels of Figs. 5 and 6. They should be compared with the low-SNR evaluation results in the middle panels of the same figures. The variance of exp3 (dotted line in the lowest left panel of Fig. 5) is slightly misleading, because further investigation of the averaged lag profiles revealed that the profile was biased due to F-region echoes.

There is one clear difference between the self-noise effects in the experiments with uniform IPPs and the PPATC experiment. This is clearly visible when comparing the continuous grey (exp1) and black (PPATC) curves in the top and bottom panels of Fig. 5. The strongest echoes and the largest self-noise contamination in the data are known to be located between $100 \mathrm{~km}$ and $300 \mathrm{~km}$. The bottom part of the grey curve (exp1) in the bottom panel of Fig. 5 is clearly higher than the corresponding curve in the middle panel of the same figure. However, the larger ranges are not much affected due to a much lower SNR. The behaviour of the continuous black curve (PPATC) is quite different: the curve has shifted upward at all ranges, and only small differences in the rate of increase are seen. The self-noise contamination of the PPATC experiment is spread over all ranges, because there are always several pulses inside the target ranges. Most of the time, one of the pulses is inside the strong ionisation, increasing the self-noise. On the other hand, the range interval where the strong ionisation is located has suffered much less in the PPATC experiment than in exp1.

In the case of pulse-to-pulse lags, the PPATC experiment seems to suffer more from the self-noise than exp3. This is due to its long pulses which spread the self-noise contamination from the E-region down to the upper D-region ranges.

\section{Test run of the PPATC experiment}

The PPATC experiment introduced in this paper was tested with the EISCAT VHF radar on 28 November 2008. Both attenuated transmitter samples and the ionospheric echoes were recorded as raw voltage data, which were analysed using lag profile inversion. In the analysis, the data was filtered and decimated to a final $15 \mu$ s sampling interval. All possible lag values from $15 \mu$ s to $10 \mathrm{~ms}$ were analysed with a five-second time resolution. The possibilities of integrating several pulse-to-pulse lags into one profile and limiting the pulse-to-pulse analysis below some maximum range smaller than the total coverage of the experiment were not used, to point out that the analysis works well also without these options.

An example of the real part of an ACF is plotted in Fig. 7. The result is not shown in absolute units, because neither the exact attenuation of the transmitter samples nor the actual transmitter power are recorded with the present sampling 
equipment. The plotted $\mathrm{ACF}$ is an average of 60 inversion results, i.e. five minutes in time. A few ACFs clearly containing coherent echoes from hard targets were removed before the averaging. The ACF reveals ionisation in E- and F-regions. The altitude profile of the ionisation is in good agreement with the simultaneous results of the Troms $\emptyset$ dynasonde. A D-region is also observed with the IS radar, the long correlations in the D-region signal are clearly visible over several milliseconds in the lowest parts of the pulse-to-pulse lag profiles.

\section{Discussion}

The benefit of the PPATC method is the possibility of measuring also the pulse-to-pulse correlations from the same data, which provides the E- and F-region autocorrelation functions. In addition to the D-region analysis, the pulse-topulse coherence is beneficial for the analysis of weak coherent echoes, e.g. from space debris and meteors. The possibility of measuring these secondary data products also makes the experiment truly multipurpose. Debris and meteor detections can also improve the plasma parameter estimation, because these coherent echoes can then be removed from the data already before the lag profile inversion step. The atmospheric echoes from low altitudes, well below the D-region, are also an interesting long-correlating target, though we do not yet have methods for separating the atmospheric signal from the very strong ground-clutter signal.

The analysis of space debris is more feasible with nonuniform IPPs, because the achievable continuous range is much larger than that achievable with uniform IPPs (Vierinen et al., 2009). Space debris is typically detected between 200 and $2500 \mathrm{~km}$, but sometimes space objects can be observed at ranges up to tens of thousands of kilometers. While the PPATC experiment described in this study can provide a continuous range coverage up to $1000 \mathrm{~km}$, it can be easily increased up to tens of thousands of kilometers, e.g. using longer simple difference covers, arithmetic modulus type aperiodic transmission sequences (Uppala and Sahr, 1994), or with a ramped IPP scheme (Vierinen et al., 2009).

If only the severely overspread targets are of interest, exactly the same range and lag coverages can be achieved by transmitting the $\mathrm{N}$ pulses in the ATC code in $\mathrm{N}$ separate frequency channels, which in addition leads to the simple situation of a constant pulse-repetition frequency in each channel. Experiments very similar to this have been in routine use at least in the EISCAT Svalbard radar for several years. We did not fully investigate the self-noise effects, but it is obvious that an experiment using several frequency channels does not suffer from the self-noise as much as the true PPATC version.

In this paper the rather complicated range ambiguities produced by the PPATC method were removed with lag profile inversion (Virtanen et al., 2008a). The analysis should be possible, at least with minor changes to the actual software, with any other method independent of any special decoding properties of the applied codes. These methods include lag profile deconvolution methods similar to the one used in this paper (Nikoukar et al., 2008), as well as the so-called fullprofile analysis (Lehtinen et al., 1996; Hysell et al., 2008b).

Despite the possibility of measuring both intra-pulse and pulse-to-pulse lags with a large range coverage, the PPATC experiment evaluated in this paper still contains gaps in the lag coverage. The most troublesome gap is evidently the transition from the longest intra-pulse lag to the shortest pulse-to-pulse lag in the E-region. The shortest IPP cannot be made short enough for two reasons: the reception cannot be started immediately after the end of a pulse because of hardware limitations plus ground clutter and, secondly, the present radar system does not allow the pulses to be transmitted closer than we are already doing. If the gap causes serious problems, the experiment could possibly be improved by combining pulses of different lengths in the same experiment, as done in Virtanen et al. (2008b). This kind of further fine-tuning of the experiments is not included in this paper, as the present goal is only to demonstrate the basic idea of the method and to show that good estimation accuracies can be achieved.

The true purpose of incoherent scatter measurements is estimating ionospheric plasma parameters, a part of the analysis which was not included in this paper. However, the accuracy of the ACF estimation was carefully studied, and the PPATC method was shown to be capable of producing ACF estimates with an accuracy near to the theoretical optimum at all lag values. Thus, the question is only about designing an experiment providing sufficient lags with high enough radar efficiency from each range. According to Vallinkoski (1989), the longest lag required for a proper parameter fit is approximately the second zero-crossing of the ACF. A natural choice would be to use long enough pulses to get the necessary lag coverage for F-region from the intra-pulse lags. As seen from Fig. 7, this requirement is hardly fulfilled with the tested experiment, and somewhat longer pulses should most probably be used in future experiments.

The ultimate goal of experiment optimisation is to find an experiment, which can be shown to be impossible to improve for all the lag profiles necessary at all the different layers of the ionosphere. As a conclusion, the PPATC is very close to this goal and one has good reasons to think that no other experiments are ever necessary, unless only a limited range interval sufficiently far away from the radar is of interest. However, one must remember that two reservations still remain, 1) the first one about theoretical understanding of accuracy limits to experiment variances with good SNR and 2) the second one dealing with the optimal bit length being determined by the desired range resolution. We discuss these below.

1. The inverse of the radar efficiency represents a theoretical limit to the normalised lag profile variance only in 
cases where the receiver thermal noise background is the dominant noise contribution, i.e. the SNR is low. If this is not the case, the self-noise causes complicated effects, including correlations between different lag profile estimates, which may lead into rather surprising results. Experiment optimisation remains an open question in this case and for example the numerical case studies in Huuskonen and Lehtinen (1996) and Lehtinen et al. (1997) show that taking these correlations into account may significantly affect the lag profile and consequently ionospheric parameter estimation variances. In some cases the variances may actually be smaller, and in some cases larger, than simple approximations would imply. Thus, in cases of good or high SNR some other experiment might be better than the PPATC. However, the simulations shown in this paper for high SNR show that no catastrophic increase of variance seem to result by use of the PPATC method. Thus at least as long as no concrete alternatives have been found, we recommend use of PPATC even in the case of better SNR.

2. We have also taken a certain range resolution as granted. In Lehtinen (1989) it is shown that in a situation with a low SNR, the desired target model resolution determines the optimal bit length of an alternating code or any other code close to the theoretical optimum in such a way that the best bit length is approximately 1.5 times the range resolution of the analysis model. As typical range resolutions in different ionospheric layers are different, this is another fact which makes it impossible to design an experiment which is simultaneously optimal for all possible purposes. If the resolution requirement for the different layers were the same, our considerations here would show that the PPATC is very close to a general-purpose optimal experiment for moderate or low SNR, but now, as different resolution requirements for different layers dictate, one needs to choose the bit length to be compatible with the resolution requirement of the most important layer and then be satisfied with the penalty caused by non-matching resolution requirements for the other layers. This fact can actually be shown to be a mathematical law, which no further code development can be expected to eliminate.

\section{Conclusions}

The utilisation of aperiodic transmitter coding for incoherent scatter radar experiments using long phase-coded pulses was demonstrated. A PPATC experiment was designed and evaluated, revealing that estimation accuracies near the theoretical optimum can be achieved when optimised phase-code sequences are used in a PPATC experiment. The designed experiment was also tested with a real radar and the data was successfully analysed with lag profile inversion.
The method can be beneficial especially in D-region studies or as a flexible all-around experiment, as it provides a method for combining a high pulse-to-pulse lag resolution with a large continuous range coverage. These kinds of experiments can also be used for space debris and meteor studies, making them truly multipurpose. This capability also significantly improves outlier removal due to these kinds of non-IS targets.

We have also shown that at least for low SNR cases, a collection of such experiments with different baud lengths might be regarded as the ultimate suite of experiments for measuring all ionospheric layers simultaneously. The question of experiment optimisation is then reduced to just the question of choosing the baud length which best corresponds to the desired resolution. While proven for low SNR only, our simulation results show that the behaviour is at least decent for good SNR, too.

Acknowledgements. This work was supported by the Academy of Finland (application number 213476, Finnish Programme for Centres of Excellence in Research 2006-2011). Ilkka Virtanen is funded by the Finnish Graduate School in Astronomy and Space Physics. The EISCAT measurements were made with special programme time granted to Finland. EISCAT is an international association supported by China (CRIRP), Finland (SA), Germany (DFG), Japan (STEL and NIPR), Norway (NFR), Sweden (VR) and United Kingdom (STFC). The authors are grateful for M. Vallinkoski for his help in preparing the manuscript, especially for his advice concerning the English language.

Topical Editor M. Pinnock thanks one anonymous referee for her/his help in evaluating this paper.

\section{References}

Chau, J. L., Hysell, D. L., Reyes, P. M., and Milla, M. A.: Improved spectral observations of equatorial spread $\mathrm{F}$ echoes at Jicamarca using aperiodic transmitter coding, J. Atmos. Solar-Terr. Phys., 66, 1543-1548, doi:10.1016/j.jastp.2004.07.002, 2004.

Clinger, W. and Van Ness, J. W.: On unequally spaced time points in time series, The Annals of Statistics, 4, 736-745, 1976.

Damtie, B., Nygrén, T., Lehtinen, M. S., and Huuskonen, A.: High resolution observations of sporadic-E layers within the polar cap ionosphere using a new incoherent scatter radar experiment, Ann. Geophys., 20, 1429-1438, 2002,

http://www.ann-geophys.net/20/1429/2002/.

Farley, D. T.: Incoherent scatter correlation function measurements, Radio Sci., 4, 935-953, 1969.

Farley, D. T.: Multiple-pulse incoherent-scatter correlation function measurements, Radio Sci., 7, 661-666, 1972.

Huuskonen, A. and Lehtinen, M. S.: The accuracy of incoherent scatter measurements: error estimates valid for high signal levels, J. Atmos. Terr. Phys., 58, 453-463, 1996.

Hysell, D. L. and Chau, J. L.: Interpreting the Doppler spectrum of coherent scatter from topside equatorial spread F, J. Atmos. Solar-Terr. Phys., 66, 1549-1557, doi:10.1016/j.jastp.2004.07. 003, 2004.

Hysell, D. L., Chau, J. L., and Lakshmanan, S.: Improved spectral estimation of equatorial spread $\mathrm{F}$ through aperiodic pulsing 
and Bayesian inversion, Radio Sci., 43, RS2010, doi:10.1029/ 2007RS003790, 2008a.

Hysell, D. L., Rodrigues, F. S., Chau, J. L., and Huba, J. D.: Full profile incoherent scatter analysis at Jicamarca, Ann. Geophys., 26, 59-75, 2008b, http://www.ann-geophys.net/26/59/2008/.

Lehtinen, M. S., Huuskonen, A., and Markkanen, M.: Randomization of alternating codes: Improving incoherent scatter measurements by reducing correlations of gated autocorrelation function estimates, Radio Sci., 32, 2271-2282, doi:10.1029/97RS02556, 1997.

Lehtinen, M. S.: On optimization of incoherent scatter measurements, Adv. Space Res., 9, 133-141, doi:10.1016/02731177(89)90351-7, 1989.

Lehtinen, M. S. and Häggström, I.: A new modulation principle for incoherent scatter measurements, Radio Sci., 22, 625-634, 1987.

Lehtinen, M. S., Huuskonen, A., and Pirttilä, J.: First experiences of full-profile analysis with GUISDAP, Ann. Geophys., 14, 14871495, 1996, http://www.ann-geophys.net/14/1487/1996/.

Lehtinen, M. S., Virtanen, I. I., and Vierinen, J.: Fast comparison of IS radar code sequences for lag profile inversion, Ann. Geophys., 26, 2291-2301, 2008, http://www.ann-geophys.net/26/2291/2008/.

Markkanen, M. and Nygrén, T.: Long alternating codes 2. Practical search method, Radio Sci., 32, 9-18, doi:10.1029/96RS03117, 1997.

Nikoukar, R., Farzad, K., and Kudeki, E.: An efficient near-optimal approach to incoherent scatter radar parameter estimation, Radio Sci., 43, RS5007, doi:10.1029/2007RS003724, 2008.

Nygrén, T. and Markkanen, M.: Long alternating codes 1 . Search by playing dominoes, Radio Sci., 32, 1-8, doi:10.1029/96RS03118, 1997.

Nygrén, T., Huuskonen, A., and Pollari, P.: Alternating-coded multipulse codes for incoherent scatter experiments, J. Atmos. Terr. Phys., 58, 465-477, 1996.

Orispää, M. and Lehtinen, M. S.: Fortran Linear Inverse Problem Solver (FLIPS), Inverse Problems and Imaging, in review, 2009.

Pirttilä, J., Lehtinen, M. S., Huuskonen, A., and Markkanen, M.: A Proposed Solution to the Range-Doppler Dilemma of Weather Radar Measurements by Using the SMPRF Codes, Practical Results, and a Comparison with Operational Measurements, J. Appl. Meteor., 44, 1375-1390, 2005.
Raizada, S., Sulzer, M. P., Tepley, C. A., Gonzalez, S. A., and Nicolls, M. J.: Inferring D region parameters using improved incoherent scatter radar techniques at Arecibo, J. Geophys. Res. (Space Physics), 113, A12302, doi:10.1029/2007JA012882, 2008.

Schleher, D. C.: MTI and Pulsed Doppler Radar, Artech House, Inc., 1991.

Skolnik, M. I. (Ed.): Radar Handbook, McGraw-Hill Publishing Company, 2nd edn., 1990.

Sulzer, M. P.: A phase modulation technique for a sevenfold statistical improvement in incoherent scatter data-taking, Radio Sci., 21, 737-744, 1986a.

Sulzer, M. P.: A radar technique for high range resolution incoherent scatter autocorrelation function measurements utilizing the full average power of klystron radars, Radio Sci., 21, 1033-1040, 1986b.

Sulzer, M. P.: Recent incoherent scatter techniques, Adv. Space Res., 9, 153-162, doi:10.1016/0273-1177(89)90353-0, 1989.

Sulzer, M. P.: A new type of alternating code for incoherent scatter measurements, Radio Sci., 28, 995-1002, 1993.

Uppala, S. V. and Sahr, J. D.: Spectrum estimation of moderately overspread radar targets using aperiodic transmitter coding, Radio Sci., 29, 611-623, 1994.

Uppala, S. V. and Sahr, J. D.: Aperiodic transmitter waveforms for spectrum estimation of moderately overspread targets: new codes and a design rule, IEEE Trans. Geosci. Rem. Sens., 34, 1285-1287, doi:10.1109/36.536545, 1996.

Vallinkoski, M.: Error Analysis of Incoherent Scatter Radar Measurements, PhD thesis, University of Helsinki, 1989.

Vierinen, J., Lehtinen, M. S., Orispää, M., and Virtanen, I. I.: Transmission code optimization method for incoherent scatter radar, Ann. Geophys., 26, 2923-2927, 2008, http://www.ann-geophys.net/26/2923/2008/.

Vierinen, J., Lehtinen, M. S., Markkanen, J., and Virtanen, I. I.: Measuring space debris with phase coded aperiodic transmission sequences, in: Proceedings of the Fifth European Conference on Space Debris, Darmstadt, Germany, 30 March-2 April 2009, ESA/ESOC, 2009.

Virtanen, I. I., Lehtinen, M. S., Nygrén, T., Orispää, M., and Vierinen, J.: Lag profile inversion method for EISCAT data analysis, Ann. Geophys., 26, 571-581, 2008a, http://www.ann-geophys.net/26/571/2008/.

Virtanen, I. I., Lehtinen, M. S., and Vierinen, J.: Towards multipurpose IS radar experiments, Ann. Geophys., 26, 2281-2289, 2008b, http://www.ann-geophys.net/26/2281/2008/. 